\title{
The stellar Mass-to-Light ratio in disc galaxies
}

\author{
L. Portinari and J. Sommer-Larsen \\ Theoretical Astrophysics Center \\ Juliane Maries Vej 30, DK-2100 Copenhagen Ø, Denmark
}

R. Tantalo

Dipartimento di Astronomia, Università di Padova

Vicolo dell'Osservatorio 2, I-35122 Padova (PD), Italy

\begin{abstract}
The mass and distribution of baryons in galaxies is closely related to the inferred structure of the dark halo. A robust estimate of the baryon content requires knowledge of the typical mass-to-light $(\mathrm{M} / \mathrm{L})$ ratio of the stellar component. Various arguments favour a low $\mathrm{M}_{*} / \mathrm{L}_{I}<1$ for late-type spirals. We show that "bottom-light" Initial Mass Functions (IMFs) favoured by recent literature do yield so low M/L ratios, for $\mathrm{Sbc} / \mathrm{Sc}$ discs. We also predict the stellar $\mathrm{M} / \mathrm{L}$ ratio, and hence the zero-point of the Tully-Fisher relation, to vary significantly with Hubble disc type. Finally, we provide colour-M/L relations to derive the stellar M/L ratio from the observed photometry, for different IMFs.
\end{abstract}

A number of dynamical arguments, as well as the brightness of the observed Tully-Fisher relation when compared to the estimated stellar mass in the Milky Way or to cosmological simulations of disc galaxy formation, favour a low $\mathrm{M}_{*} / \mathrm{L}_{I}=0.7-1$ for late-type spirals (Portinari et al. 2003; hereinafter PST).

In PST we studied the stellar $\mathrm{M} / \mathrm{L}$ ratio in disc galaxies, with chemophotometric models adopting different formulations for the Initial Mass Function (IMF): the classic Salpeter (1955) IMF and a number of "bottom-light" IMFs characterized by less mass stored in low-mass, low-luminosity stars (Kroupa 1998; Kennicutt et al. 1994; Larson 1998; Chabrier 2001, 2002). While the Salpeter IMF results in far too high $\mathrm{M} / \mathrm{L}$ ratios, the Kroupa IMF is just slightly too "heavy" and all the other bottom-light IMFs yield $\mathrm{M}_{*} / \mathrm{L}_{I}<1$ for Sbc/Sc discs (corresponding to a "star formation history parameter" $b=0.8-1$ in Fig. 1).

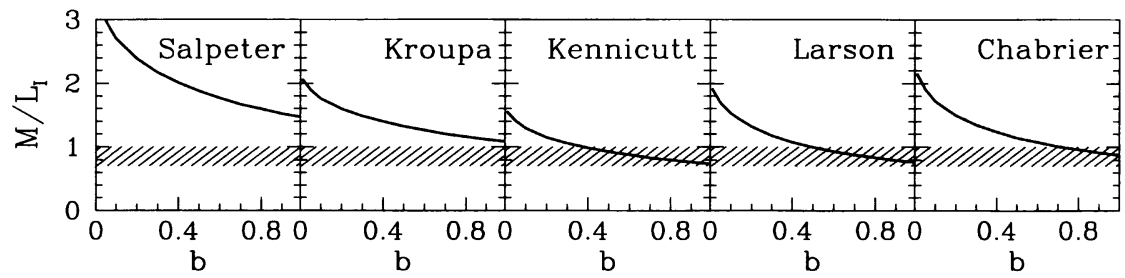

Figure 1. I-band stellar M/L ratio at varying $b$ parameter of the star formation history. The shaded area marks the range $M / L_{I}$ favoured by observations for $\mathrm{Sbc} / \mathrm{Sc}$ spirals (corresponding to $b=0.8-1$ ). 

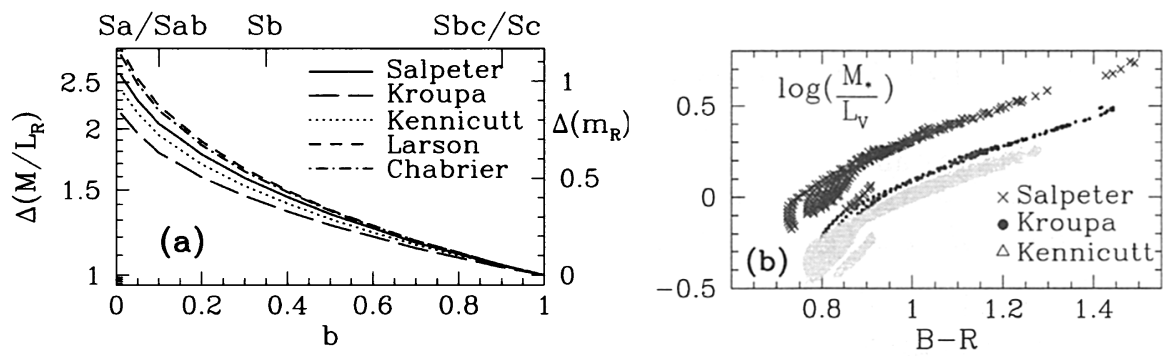

Figure 2. (a) Relative $\mathrm{M} / \mathrm{L}$ ratio in the $\mathrm{R}$-band, normalized to that of $b=1$ models. (b) Example of colour $-\mathrm{M} / \mathrm{L}$ ratio relations.

Offsets in the Tully-Fisher relation with Hubble type. The stellar M/L ratio depends on the IMF but also on the star formation history of the galaxy, typically parameterized by $b=S F R /\langle S F R\rangle$, the ratio between the presentday and the average past Star Formation Rate. The Hubble sequence of galaxies is also a colour sequence, hence a sequence in characteristic star formation histories: $b \sim 0.1$ for Sa/Sab spirals, $b \sim 0.35$ for Sb, and $b \sim 0.8-1$ for Sbc/Sc (Kennicutt et al. 1994; Sommer-Larsen et al. 2003). Lower $b$-parameters correspond to an increasing percentage of old stellar populations, hence to an increasing stellar M/L ratio (Fig. 1). This results in systematic offsets for the luminosity zero-point of the Tully-Fisher relation. With respect to Sbc/Sc spirals, Sb's are expected to be 0.3-0.4 mag dimmer and Sa/Sab's 0.6-0.8 mag dimmer in the red optical bands (Fig. 2a), in good agreement with the empirical offsets determined by Kannappan et al. (2002).

Colour $-\mathbf{M} / \mathbf{L}$ ratio relations. Colours are a probe of the star formation history of a galaxy, hence there is a tight correlation between the stellar $\mathrm{M} / \mathrm{L}$ ratio and colours (Fig. 2b; Bell \& de Jong 2001). To a first approximation, the IMF sets the zero-point of the $\mathrm{M} / \mathrm{L}$ ratio, while the colour dependence (slope of the relation in Fig. 2b) is the same for all IMFs. In PST (Appendix B) we provide theoretical relations to infer the stellar $\mathrm{M} / \mathrm{L}$ ratio from the observed colours, once an IMF is assumed.

\section{References}

Bell E.F. \& de Jong R.S., 2001, ApJ, 550, 212

Chabrier G., 2001, ApJ, 554, 1274

Chabrier G., 2002, ApJ, 567, 304

Kannappan S.J., Fabricant D.G. \& Franx M., 2002, AJ, 123, 2358

Kennicutt R.C., Tamblyn P. \& Congdon C.W., 1994, ApJ, 435, 22

Kroupa P., 1998, in ASP Conf. Ser. Vol. 134, p. 483

Larson R.B., 1998, MNRAS, 301, 569

Portinari L., Sommer-Larsen J. \& Tantalo R., 2003, MNRAS, in press (astro$\mathrm{ph} / 0303190)$ (PST)

Salpeter E.E., 1955, ApJ, 121, 161

Sommer-Larsen J., Götz M. \& Portinari L., 2003, ApJ, 596, 47 